\title{
LOCAL ASYMPTOTIC DISTRIBUTION OF ZEROS OF ORTHOGONAL POLYNOMIALS
}

\author{
VILMOS TOTIK AND JOSEPH L. ULLMAN
}

\begin{abstract}
Converse results, which state a relation (inequality) for measures from that on their logarithmic potentials, are applied to local density of zeros of orthogonal polynomials when the measure of orthogonality is a general one with compact support. It will be shown that if the measure is sufficiently thick on a part of its support, then on that part the density of the zeros will be at least as large as the equilibrium measure of the support. A corresponding upper estimate on the distribution of the zeros will also be proved. All of our estimates are sharp, and they localize several well-known results.
\end{abstract}

\section{INTRODUCTION}

The aim of the present work is to get estimates on the local density of zeros of orthogonal polynomials when the generating measure is a general one with compact support which has empty interior and connected complement. For example, the results can be applied to any measure supported on the real line. The main objective will be to show that if the measure of orthogonality is sufficiently thick on a part of its support, then on that part the density of the zeros will be at least as large as the equilibrium measure of the support. This localizes several classical theorems. We shall also prove a corresponding upper estimate on the distribution of the zeros. Both estimates will turn out to be sharp.

The method of the paper is potential theoretical. In fact, the results concerning local density of zeros will be derived from a theorem due to De la Vallée Poussin [dVP2] which states an inequality for measures from that on their logarithmic potentials. In the literature this theorem has been rediscovered several times (see the discussion in the next section) in slightly different forms.

The paper is organized as follows. In the next section we shall present the necessary potential theoretical background for the whole paper. We will also discuss the above-mentioned converse results on measures and potentials that will be the key to all of the later proofs. The applications concerning general orthogonal polynomials will be given in $\S 3.1$. First we will give the necessary background, then state the theorems, and the actual proofs will be given in $\S 3.2$.

Received by the editors July 22, 1991 and, in revised form, January 28, 1992.

1991 Mathematics Subject Classification. Primary 42C05, 31A05.

Research of the first author was supported by NSF Grant No. DMS 9002794. 


\section{BACKGROUNDS AND CONVERSE RESULTS FOR MEASURES AND LOGARITHMIC POTENTIALS}

2.1. Potential theoretical backgrounds. We shall be working with Borel measures with compact support on $\mathbf{C}$. The logarithmic potential of such a measure $\nu$ is defined as the convolution with the logarithmic kernel:

$$
U^{\nu}(z):=\int \log \frac{1}{|z-t|} d \nu(t) .
$$

The logarithmic energy of $\nu$ is given as

$$
I(\nu):=\int U^{\nu}(z) d \nu(z)=\iint \log \frac{1}{|z-t|} d \nu(t) d \nu(z) .
$$

If $K$ is a compact set, then its logarithmic capacity $\operatorname{cap}(K)$ is defined by the formula

$$
\log \frac{1}{\operatorname{cap}(K)}:=\inf \{I(\nu) \mid \operatorname{supp}(\nu) \subset K,\|\nu\|=1\},
$$

where $\|\nu\|$ denotes the total variation (total mass) of $\nu$. Now the capacity of an arbitrary Borel set $B$ is defined as the supremum of the capacities of compact subsets of $B$, and a property is said to hold quasi-everywhere on a set $A$ if it holds at every point of $A$ with the exception of points of a set of capacity zero.

We shall also need the existence of the equilibrium measure for a Borel subset of C (see [dVP1], [Wy] or [StTo, Appendix IV]): If $E$ is a bounded Borel subset of $\mathbf{C}$ of positive capacity, then there exists a unique probability measure $\omega_{E}$ with the following properties:

$$
\text { (ii) } \quad U^{\omega_{E}}(z)=\log \frac{1}{\operatorname{cap}(E)} \text { for quasi-every } z \in E \text {. }
$$

In the case when $E$ is compact, the equilibrium measure $\omega_{E}$ is supported on $E$ (more precisely on its outer boundary, which is defined as the boundary of the unbounded connected component of $\mathbf{C} \backslash E$ ). In the general case we only can assert that $\operatorname{supp}\left(\omega_{E}\right)$ is contained in the closure of $E$.

2.2. Two inverse results on measures and potentials. Our proofs will be based on

Theorem 1. Let $\mu$ and $\nu$ be two measures of compact support, and let $\Omega$ be a domain in which both potentials $U^{\mu}$ and $U^{\nu}$ are finite and satisfy with some constant $c$ the inequality

$$
U^{\mu}(z) \leq U^{\nu}(z)+c, \quad z \in \Omega .
$$

If $A$ is the subset of $\Omega$ in which equality holds in (3), then $\left.\nu\right|_{A} \leq\left.\mu\right|_{A}$, i.e., for every Borel subset $B$ of $A$ the inequality $\nu(B) \leq \mu(B)$ holds.

Let us note that the finiteness of the potentials in question is necessary, as can be seen by the example of the unit disc as $\Omega$ and $\mu=\delta_{0}, \nu=2 \delta_{0}$ as the two measures, where $\delta_{0}$ denotes the 'Dirac delta' mass concentrated at 0 . 
Theorem 1 is a special case of a result of De la Vallée Poussin [dVP2] (in fact, it is equivalent to it), who did not require the finiteness of the potentials in $\Omega$, instead considered the set $A$ where both potentials are finite and the equality holds. Brelot $[\mathrm{Br}]$ had a more general version, and Grishin $[\mathrm{Gr}]$ considered a larger set $A$ where the stated inequality still holds. Finally, an even more general result, in which the "equality set" $A$ is defined as the equality set in the fine topology, will be published by Fuglede [Fu]. Since the rest of the present paper is based on Theorem 1, and at this moment there is no published proof of it in English, for completeness we include a rather elementary proof. For further discussion and examples concerning related results we refer to [Fu].

Theorem 1 is of local character, now we discuss a global variant.

Theorem 2. Let $\nu$ and $\sigma$ be finite measures of compact support such that $\|\nu\| \geq$ $\|\sigma\|$, and assume that the potential $U^{\sigma}$ of $\sigma$ is finite everywhere. If for some constant $c$ we set

$$
H:=\left\{z \mid U^{\nu}(z)>U^{\sigma}(z)+c\right\},
$$

then

$$
\nu(H) \geq \sigma(H) .
$$

If the set in (4) is nice, say it is open and its boundary has continuous inner normal $\mathbf{n}$, then in some cases (5) can be immediately deduced from Gauss' formula: if $H$ is a bounded open set with smooth boundary and $\tau$ is a signed measure of compact support in $H$, then

$$
\tau(H)=\frac{1}{2 \pi} \int_{\partial H} \frac{\partial U^{\tau}}{\partial \mathbf{n}} .
$$

However, in the general case some caution is necessary, as the conclusion in the theorem may fail if we drop the assumptions.

As a matter of fact, Theorems 1 and 2 are equivalent. They are not as special as they seem at first glance, in fact, in many situations it is relatively easy to transform the measures in the problem into related ones for which either Theorem 1 or 2 can be applied. We shall apply such a technique to deduce Theorem 2 from Theorem 1.

2.3. Proof of Theorems 1 and 2. To prove Theorem 1 first we need a definition and a theorem from [Be] which we put into a vocabulary consistent with this paper.

Let $B$ be a set in the plane and let $\Gamma$ be a set of open discs such that to each $z$ of $B$ there are discs of $\Gamma$ with center $z$ and of arbitrarily small positive radii. We say that then $\Gamma$ covers $B$ in the Vitali narrow sense.

Lemma 1. Let $\mu$ be a finite Borel measure in the plane and let $B$ be a bounded Borel set such that $\mu(\mathbf{C} \backslash B)=0$. If $\Gamma$ is a set of discs which covers $B$ in the Vitali narrow sense, then $\Gamma$ contains a countable subset $\bar{\Gamma}=\left\{\Delta_{i}\right\}$ of nonoverlapping discs such that $\mu(B)=\mu\left(\bigcup \Delta_{i}\right)$.

The proof of Theorem 1 is now based on the following fact: Let $A$ be the set defined in Theorem 1. Then there is a set of discs $\Gamma$ which covers $A$ in the 
Vitali narrow sense and is such that for $\mu, \nu$ as introduced in this Theorem, if $\Delta \in \Gamma$, then

$$
\nu(\Delta) \leq \mu(\Delta) .
$$

We postpone the poof of this fact and first show how to deduce Theorem 1 from this fact.

Let $B$ be a Borel subset of the set $A$ and let $\Gamma$ be the set of discs associated with $A$ as guaranteed above. Let $\epsilon>0$ be an arbitrary constant and let $O$ be an open set containing $B$ such that $\mu(O) \leq \mu(B)+\epsilon$. Let $\Gamma_{1}$ consist of all of the discs of $\Gamma$ which lie in $O$. We then see that $\Gamma_{1}$ covers $B$ in the Vitali narrow sense. Let $\nu_{1}$ be the measure $\nu$ restricted to $B$. We then have, by Lemma 1 a countable collection of discs of $\Gamma_{1}$, say $\bar{\Gamma}_{1}=\left\{\Delta_{i}\right\}$, which are nonoverlapping, and satisfy $\nu_{1}\left(\bigcup \Delta_{i}\right)=\nu_{1}(B)$. We then have

$$
\begin{aligned}
\nu(B) & =\nu_{1}(B)=\nu_{1}\left(\bigcup \Delta_{i}\right)=\sum \nu_{1}\left(\Delta_{i}\right) \leq \sum \nu\left(\Delta_{i}\right) \\
& \leq \sum \mu\left(\Delta_{i}\right)=\mu\left(\bigcup \Delta_{i}\right) \leq \mu(O) \leq \mu(B)+\epsilon .
\end{aligned}
$$

Since this argument can be carried out for every $\epsilon>0$, we finally obtain the required inequality $\nu(B) \leq \mu(B)$.

To prove the above claim concerning the existence of a covering in the Vitali narrow sense we first state some facts concerning logarithmic potentials (see [Ts, pp. 38-49]). Let $U(z)=U^{\mu}(z)$ be a potential that is finite on a domain $G$. Then for any $z_{0} \in G$, and $r>0$ for which the disk $\Delta\left(z_{0}, r\right):=\left\{z|| z-z_{0} \mid<\right.$ $r$ \} is contained in $G$, the mean value

$$
L\left(U ; z_{0}, r\right)=\frac{1}{2 \pi} \int U\left(z_{0}+r e^{i \theta}\right) d \theta
$$

exists as a finite number, and $L\left(U ; z_{0}, r\right)$ is a nonincreasing function of $r$ that is absolutely continuous on any closed subinterval of $(0, \infty)$. Furthermore,

$$
\lim _{r \rightarrow 0} L\left(U ; z_{0}, r\right)=U\left(z_{0}\right)
$$

If $r$ is a value for which $\frac{d}{d r} L\left(U ; z_{0}, r\right)$ exists, then $\Delta\left(z_{0}, r\right)$ is called regular disc, and for a regular disc

$$
\mu\left(\Delta\left(z_{0}, r\right)\right)=-r \frac{d}{d r} L\left(U ; z_{0}, r\right) .
$$

Now let $A$ be the set from Theorem 1 , and let $z_{0}$ be any point in $A$. We want to prove that there is a sequence $r_{n}=r_{n}\left(z_{0}\right), r_{n}>0, \lim _{n \rightarrow \infty} r_{n}=0$ such that

$$
\nu\left(\Delta\left(z_{0}, r_{n}\right)\right) \leq \mu\left(\Delta\left(z_{0}, r_{n}\right)\right)
$$

The collection

$$
\Gamma=\left\{\Delta\left(z_{0}, r_{n}\left(z_{0}\right)\right) \mid z_{0} \in A, n=1,2, \ldots\right\}
$$

then covers $A$ in the Vitali narrow sense and has the desired further property (7). 
Let $U_{1}\left(z_{0}\right)=U^{\mu}\left(z_{0}\right)$ and $U_{2}\left(z_{0}\right)=U^{\nu}\left(z_{0}\right)$, and for $i=1,2$ set

$$
L_{i}\left(z_{0}, r\right)=\frac{1}{2 \pi} \int U_{i}\left(z_{0}+r e^{i \theta}\right) d \theta
$$

Now since $U_{1}(z) \leq U_{2}(z)+c$ in a neighborhood of $z_{0}$, we have $L_{1}\left(z_{0}, r\right) \leq$ $L_{2}\left(z_{0}, r\right)+c$ for every small $r>0$. For $z_{0} \in A$ the potentials $U_{i}\left(z_{0}\right), i=$ 1,2 , are finite, and $U_{1}\left(z_{0}\right)=U_{2}\left(z_{0}\right)+c$. By subtracting we get

$$
L_{1}\left(z_{0}, r\right)-U_{1}\left(z_{0}\right) \leq L_{2}\left(z_{0}, r\right)-U_{2}\left(z_{0}\right) \text {. }
$$

Since $L_{1}(z, r)$ is absolutely continuous on any $[\epsilon, R]$ we have

$$
\int_{\epsilon}^{R} \frac{d}{d s} L_{1}\left(z_{0}, s\right) d s=L_{1}\left(z_{0}, R\right)-L_{1}\left(z_{0}, \epsilon\right) .
$$

By the nonpositivity of $\frac{d}{d s} L_{1}\left(z_{0}, s\right)$, we can let here $\epsilon \rightarrow 0$ and use Lebesgue's monotone convergence theorem to obtain

$$
\int_{0}^{R} \frac{d}{d s} L_{1}\left(z_{0}, s\right) d s=L_{1}\left(z_{0}, r\right)-U_{1}\left(z_{0}\right) .
$$

Since all steps hold when the subscript 1 is replaced by 2 , we have from (10)

$$
\int_{0}^{R} \frac{d}{d s} L_{1}\left(z_{0}, s\right) d s \leq \int_{0}^{R} \frac{d}{d s} L_{2}\left(z_{0}, s\right) d s,
$$

so that for a set of positive measure on $[0, R]$ we have

$$
\frac{d}{d r} L_{1}\left(z_{0}, r\right) \leq \frac{d}{d r} L_{2}\left(z_{0}, r\right)
$$

Since this argument can be repeated for any value of $R$, however small, we get from (9) that there are $r_{n}=r_{n}\left(z_{0}\right), r_{n}>0, \lim _{n \rightarrow \infty} r_{n} \rightarrow 0$ such that

$$
\mu\left(\Delta\left(z_{0}, r_{n}\right) \geq \nu\left(\Delta\left(z_{0}, r_{n}\right)\right),\right.
$$

which completes the proof of the claim about the existence of the cover in the Vitali narrow sense with property (7).

After this we turn to the proof of Theorem 2. By replacing $\nu$ by $\nu+\epsilon \omega_{\Delta}$, where $\omega_{\Delta}$ denotes the equilibrium measure of the unit disc $\Delta$, and then letting $\epsilon>0$ go to zero we can assume that $\|\nu\|>\|\sigma\|$. Then $H$ is bounded.

Consider the superharmonic function

$$
s(z)=\min \left\{U^{\nu}(z), U^{\sigma}(z)+c\right\} .
$$

By the Riesz representation theorem [Ts, Theorem II.24] there is a measure $\mu$ such that on every disk $D$ the difference

$$
u_{D}(z):=s(z)-\int_{D} \log \frac{1}{|z-t|} d \mu(t)
$$

is harmonic in $D$. We also know that for large $z$ the function $s(z)$ coincides with $U^{\nu}(z)$, and the latter one is harmonic outside a closed disk $\overline{D_{0}}$. Hence, by the corollary to the Riesz representation theorem in [Ts], $\mu$ does not have mass outside $\overline{D_{0}}$. This implies first of all that $u_{D}$ coincides with a function $u$ 
harmonic on the whole plane whenever $\overline{D_{0}} \subseteq D$, and it also implies via Gauss' formula (6) that $\|\nu\|=\|\mu\|$. The last two facts yield that $u$ must tend to zero at infinity, hence $u \equiv 0$. In summary, we have shown that $s(z)$ coincides with the logarithmic potential $U^{\mu}(z)$ of $\mu$.

After this preparation the proof of Theorem 2 can be easily deduced from Theorem 1. In fact, we have $U^{\mu}(z) \leq U^{\nu}(z)$ everywhere, with equality in $\mathrm{C} \backslash H$, hence by Theorem 1 we have $\left.\nu\right|_{\mathbf{C} \backslash H} \leq\left.\mu\right|_{\mathbf{C} \backslash H}$. In particular, $\nu(\mathbf{C} \backslash H) \leq$ $\mu(\mathbf{C} \backslash H)$, which in view of $\|\nu\|=\|\mu\|$ gives

$$
\mu(H) \leq \nu(H) .
$$

We also have $U^{\mu}(z) \leq U^{\sigma}(z)+c$ with equality on $H$, and so it follows again from Theorem 1 that $\sigma(H) \leq \mu(H)$. This and the previous relation gives $\sigma(H) \leq \nu(H)$.

\section{LOCAL DENSITY OF ZEROS OF ORTHOGONAL POLYNOMIALS}

3.1. Statements of the theorems and their corollaries. Let $\mu$ be a finite Borel measure on $\mathrm{C}$ with compact support $S(\mu):=\operatorname{supp}(\mu)$. We shall always assume that the support of $\mu$ consists of infinitely many points. Then we can form the uniquely existing orthonormal polynomials

$$
p_{n}(\mu ; z)=\gamma_{n}(\mu) z^{n}+\cdots, \quad \gamma_{n}(\mu)>0, n \in \mathbf{N},
$$

with respect to $\mu$, which are defined by the orthogonality relations

$$
\int p_{n}(\mu ; z) \overline{p_{m}(\mu ; z)} d \mu(z)=\delta_{n, m}
$$

where $\delta_{n, m}=1$ if $n=m$ and $\delta_{n, m}=0$ otherwise. $\gamma_{n}(\mu)$ is called the leading coefficient of $p_{n}(\mu ; \cdot)$.

For the leading coefficients $\gamma_{n}(\mu)$ we have the asymptotic estimate [StTo, Corollary 1.1.7]

$$
\frac{1}{\operatorname{cap}(S(\mu))} \leq \liminf _{n \rightarrow \infty} \gamma_{n}(\mu)^{1 / n}
$$

When the leading coefficients are asymptotically minimal, i.e., when

$$
\lim _{n \rightarrow \infty} \gamma_{n}(\mu)^{1 / n}=\frac{1}{\operatorname{cap}(S(\mu))}
$$

holds, then the orthogonal polynomial $p_{n}(\mu ; \cdot)$ are said to have regular $n$th root asymptotic behavior, and we abbreviate this fact as $\mu \in \mathbf{R e g}$. It turns out that orthogonal polynomials with this property share many properties of classical orthogonal polynomials, e.g., we have for their zero distribution the equilibrium distribution of $\operatorname{supp}(\mu)=S(\mu)$ (see [U11-4] or [StTo, Theorem 3.1.4]): if $\nu_{n}(\mu)$ is the measure that places mass $1 / n$ to every zero of $p_{n}(\mu ; \cdot)$ (this is the so-called normalized counting measure on the zeros), then $\mu \in \mathbf{R e g}$ implies (at least in the case when $S(\mu)=\operatorname{supp}(\mu)$ has empty interior and connected complement in $\mathbf{C}$ ) 


$$
\nu_{n}(\mu) \rightarrow \omega_{S(\mu)}
$$

in the weak* topology of measures on $\mathbf{C}$. Let us mention that the converse is also true with the assumption that there is no set $E$ of capacity zero that carries $\mu$ in the sense that the complement of $E$ has zero $\mu$-measure (in the terminology to be introduced below this means that $c_{\mu}>0$ ) (see [U12] or [StTo, Theorem 2.2.1]).

Orthogonal polynomials with the property (12) are said to have regular asymptotic zero distribution. Thus, regular $n$th root asymptotic behavior implies regular asymptotic zero distribution, and the converse is also true under the assumption mentioned above (in general, however, regular asymptotic zero distribution may occur without regular $n$th root asymptotic behavior taking place).

Intuitively $\mu \in$ Reg means a certain denseness of $\mu$ on its support, e.g., discrete measures of the form $\sum c_{k} \delta_{x_{k}}$ with rapidly decreasing positive coefficients $c_{k}$ will not be regular. If, however, $\mu$ is supported on the real line, say $\operatorname{supp}(\mu)=[-1,1]$, and for almost every $x \in[-1,1]$ we have

$$
\liminf _{r \rightarrow 0} e^{\eta / r} \mu([x-r, x+r])>0 \text { for every } \eta>0,
$$

then $\mu \in \operatorname{Reg}$ [StTo, Theorem 4.2.5]. In particular, this is the case if the RadonNikodym derivative $\mu^{\prime}(x)$ of $\mu$ with respect to Lebesgue measure is positive almost everywhere on $[-1,1]$ (cf. [ErTu]).

We shall also use the regularity of $\mu$ on a set $K$. We say that $\mu$ is regular on the set $K$ if

$$
\lim _{n \rightarrow \infty} \gamma_{n}\left(\left.\mu\right|_{K}\right)^{1 / n}=\frac{1}{\operatorname{cap}(K)} .
$$

This is equivalent to $\operatorname{cap}\left(\operatorname{supp}\left(\left.\mu\right|_{K}\right)\right)=\operatorname{cap}(K)$ and $\left.\mu\right|_{K} \in \operatorname{Reg}($ see (11)).

We shall now address the problem if local density of the measure of orthogonality implies any density of the zeros. The answer is provided by the next theorem. Since we want to speak of equilibrium measures, from now on we shall assume that the support $S(\mu):=\operatorname{supp}(\mu)$ is of positive capacity.

Theorem 3. Let us suppose that $S(\mu):=\operatorname{supp}(\mu)$ is compact, has empty interior and connected complement. Let further $K$ be a compact subset of $S(\mu):=$ $\operatorname{supp}(\mu)$. If $\mu$ is regular on $K$, then for any weak ${ }^{*}$ limit $\nu$ of the normalized zero counting measures $\nu_{n}(\mu)$ we have that

$$
\left.\nu\right|_{K} \geq\left.\omega_{S(\mu)}\right|_{K} .
$$

The conclusion is an inequality between two measures, and in more detailed form it asserts that for any Borel subset $B$ of $K$ we have $\nu(B) \geq \omega_{S(\mu)}(B)$.

This theorem tells us that if the measure $\mu$ is sufficiently thick on $K$ to force the orthogonal polynomials $p_{n}\left(\left.\mu\right|_{K} ; \cdot\right)$ to have $n$th root asymptotic behavior, then on $K$ the orthogonal polynomials $p_{n}(\mu ; \cdot)$ aymptotically have $n \omega_{S(\mu)}$ zeros, which means more exactly, that if $K_{1}$ is any compact subset of $K$ and $G$ is any neighborhood of $K_{1}$, then for large $n$ in $G$ the orthogonal polynomials $p_{n}(\mu ; \cdot)$ have at least $n \omega_{S(\mu)}\left(K_{1}\right)$ zeros (in fact, for $n$ belonging to the subsequence for which the weak* limit $\nu$ is taken, this follows from Theorem 3, but since $\nu$ is arbitrary, the conclusion follows for all $n$ ). 
Let us mention that the assumption in Theorem 3 that the support have empty interior and connected complement is necessary to make the conclusion. Consider, e.g., the case of Lebesgue measure on a circle or disk. In both cases the zeros of the corresponding orthogonal polynomials are at the center of the cicle.

As an upper estimate on the density of the zeros we have

Theorem 4. With the assumptions of Theorem 3 let us suppose in addition that $K$ is of positive capacity. Then for any weak* limit $\nu$ of the normalized zero counting measures $\nu_{n}(\mu)$ we have

$$
\left.\nu\right|_{K} \leq \omega_{K}
$$

The latter inequality translated into a statement about zeros means that if $K_{1}$ is any compact subset of $K$ and $G$ is any neighborhood of $K_{1}$, then for large $n$ in $K_{1}$ the orthogonal polynomials $p_{n}(\mu ; \cdot)$ have at most $n \omega_{K}(G)$ zeros.

As an immediate corollary we state

Corollary 1. Let the measure $\mu$ be supported on $[-1,1]$, and suppose that for almost every point $x$ of the interval $[a, b] \subseteq[-1,1]$ the relation (13) holds. Then the asymptotic density of the zeros of $p_{n}(\mu ; \cdot)$ on $[a, b]$ is at least as large as

$$
\frac{1}{\pi} \frac{1}{\sqrt{1-x^{2}}} d x
$$

In particular, the conclusion holds if $\mu^{\prime}(x)>0$ almost everywhere on $[a, b]$.

The lower bound given for the density of the zeros is nothing else than the classical arcsine distribution. Note that to make the conclusion we do not assume anything on $\mu$ outside $[a, b](\operatorname{except} \operatorname{supp}(\mu) \subseteq[-1,1])$. This is the local version of the zero distribution we had in mind in the introduction.

The corresponding upper estimate reads as

Corollary 2. With the assumptions of Corollary 1 the asymptotic density of the zeros on $[a, b]$ is bounded from above by

$$
\frac{1}{\pi} \frac{1}{\sqrt{(x-a)(b-x)}} d x .
$$

Let us mention that the inequalities given in Theorems 3,4 and their corollaries are sharp, for certain measures the inequalities may become equalities. For Theorem 3 this is quite obvious, we have equality for the classical orthogonal polynomials of Jacobi, say (cf. Corollary 1). To see that neither can Theorem 4 (or Corollary 2) be sharpened, consider the measure

$$
\mu=\sigma_{[a, b]}+\sum_{i} c_{i} \delta_{x_{i}},
$$

where $\sigma_{[a, b]}$ denotes the restriction of the linear Lebesgue measue to $[a, b] \subseteq$ $[-1,1]$, and $\left\{x_{i}\right\}$ is a sequence dense in $[-1,1] \backslash[a, b]$. Then $S(\mu)=$ $[-1,1]$, and if the sequence $\left\{c_{i}\right\}$ of positive numbers tends to zero sufficiently rapidly, then the asymptotic zero distribution of the orthogonal polynomials $p_{n}(\mu ; \cdot)$ will be the same as that of $p_{n}\left(\sigma_{[a, b]} ; \cdot\right)$, and the latter one is exactly the arcsine distribution transformed to $[a, b]$, that is what is on the right side of (15). 
Now we will specify a subset of the support on which Theorems 3 and 4 can be automatically applied.

Let $\Gamma(\mu)$ be the set of all carriers of the measure $\mu$, i.e.,

$$
\Gamma(\mu):=\{C \subseteq \mathbf{C} \mid C \text { a Borel set and } \mu(\mathbf{C} \backslash C)=0\} .
$$

Then the minimal carrier capacity (of the measure $\mu$ ) is defined as

$$
c_{\mu}:=\inf \{\operatorname{cap}(C) \mid C \in \Gamma(\mu)\} .
$$

With $c_{\mu}$ the lower estimate (11) for the leading coefficients can be supplemented as

$$
\limsup _{n \rightarrow \infty} \gamma_{n}(\mu)^{1 / n} \leq \frac{1}{c_{\mu}}
$$

(see [StTo, Corollary 1.1.7]).

There are carriers, called minimal carriers, $C \in \Gamma(\mu)$ for which $\operatorname{cap}(C)=$ $c_{\mu}$. It immediately follows from the definitions that if $C_{1}$ and $C_{2}$ are two minimal carriers of $\mu$ then so is $C_{1} \cap C_{2}$, and so the equilibrium measures $\omega_{C_{1}}$ and $\omega_{C_{2}}$ are both equilibrium measures for $C_{1} \cap C_{2}$. Hence, the unicity of the equilibrium measure shows that $\omega_{C_{1}}=\omega_{C_{2}}$. Now the minimal carrier equilibrium measure $\omega_{\mu}$ (of the measure $\mu$ ) can be defined as $\omega_{\mu}:=\omega_{C}$ for some minimal carrier $C$. Naturally, this definition makes sense only if $c_{\mu}>0$. The set

$$
A(\mu):=\left\{z \mid U^{\omega_{\mu}}(z)=\log \left(1 / c_{\mu}\right)\right\}
$$

is called the adjoint set associated with $\mu$. Thus, for the minimal carrier equilibrium measure we have

$$
\begin{gathered}
U^{\omega_{\mu}}(z) \leq \log \left(1 / c_{\mu}\right), \quad z \in \mathbf{C}, \\
U^{\omega_{\mu}}(z)=\log \left(1 / c_{\mu}\right), \quad z \in A(\mu) .
\end{gathered}
$$

Our next theorem shows that Theorem 3 holds for the adjoint set.

Theorem 5. Let us suppose that $S(\mu)=\operatorname{supp}(\mu)$ is compact, has empty interior and connected complement. If $c_{\mu}>0$, then for any weak* limit $\nu$ of the normalized zero counting measures $\nu_{n}(\mu)$ we have the estimate

$$
\left.\nu\right|_{A(\mu)} \geq\left.\omega_{S(\mu)}\right|_{A(\mu)} .
$$

Although it is not easy to determine the adjoint set, this theorem has the following consequence:

Corollary 3. Let us suppose that $\operatorname{supp}(\mu)$ is compact, has empty interior and connected complement. Let us also assume that $c_{\mu}>0$, and let $C$ be any minimal carrier of $\mu$. Then for any weak ${ }^{*}$ limit $\nu$ of the normalized zero counting measures $\nu_{n}(\mu)$ we have the inequality

$$
\left.\nu\right|_{C} \geq\left.\omega_{S(\mu)}\right|_{C} .
$$

In particular, if $S$ is a subset of $S(\mu)$ such that the Radon-Nikodym derivative $d \mu(x) / d \omega_{S(\mu)}$ is positive for $\omega_{S(\mu)}$-almost every $x \in S$, then for any weak ${ }^{*}$ 
limit $\nu$ of the normalized zero counting measures $\nu_{n}(\mu)$ we have

$$
\left.\nu\right|_{S} \geq\left.\omega_{S(\mu)}\right|_{s}
$$

The corresponding upper estimate is given in

Theorem 6. With the assumptions of Theorem 5 for any weak* limit $\nu$ of the normalized zero counting measures $\nu_{n}(\mu)$ we have

$$
\left.\nu\right|_{A(\mu)} \leq \omega_{\mu} .
$$

Corollary 4. Let us suppose that $\operatorname{supp}(\mu)$ is compact, has empty interior and connected complement. Let us also assume that $c_{\mu}>0$, and let $C$ be any minimal carrier of $\mu$. Then for any weak ${ }^{*}$ limit $\nu$ of the normalized zero counting measures $\nu_{n}(\mu)$ we have the inequality

$$
\left.\nu\right|_{C} \leq \omega_{\mu} .
$$

\subsection{Proofs.}

3.2.1. Proof of Theorems 3 and 5. Since the measure $\omega_{S(\mu)}$ has finite logarithmic energy, sets of capacity zero have zero $\omega_{S(\mu)}$-measures, so without loss of generality we can assume that $K$ has positive capacity, otherwise the statement of Theorem 3 is trivial.

We begin with the following limit relations on the orthonormal polynomials: if the support $S(\mu)$ of $\mu$ has empty interior and connected complement, then for every infinite subsequence $N$ of the natural numbers

$$
\begin{aligned}
\exp \left(\log \frac{1}{\operatorname{cap}(S(\mu))}-U^{\omega_{S(\mu)}}(z)\right) & \leq \limsup _{n \rightarrow \infty, n \in N}\left|p_{n}(\mu ; z)\right|^{1 / n} \\
& \leq \exp \left(\log \frac{1}{c_{\mu}}-U^{\omega_{\mu}}(z)\right)
\end{aligned}
$$

for quasi-every $z \in \mathbf{C}$ (see [StTo, Theorem 1.1.4; Ul1, U12]), where $c_{\mu}$ and $\omega_{\mu}$ denote the minimal carrier capacity and minimal carrier equilibrium measure corresponding to $\mu$ as was defined in $\S 3.1$.

Now we need the principle of descent according to which if $\nu_{n}$ is a sequence of measures all having support in a fixed compact subset of $\mathbf{C}$ and if $\nu_{n} \rightarrow \nu$ in the weak ${ }^{*}$ topology of measures, then for every $z \in \mathbf{C}$

$$
U^{\nu}(z) \leq \liminf _{n \rightarrow \infty} U^{\nu_{n}}(z) .
$$

On the other hand, the lower envelope theorem says that in (20) the equality sign holds for quasi-every $z \in \mathbf{C}$.

The relation of polynomials with logarithmic potentials is immediate from the formula

$$
\frac{1}{n} \log \frac{1}{\left|p_{n}(\mu ; z)\right|}=\log \frac{1}{\gamma_{n}(\mu)^{1 / n}}+U^{\nu_{n}}(z),
$$

where $\nu_{n}$ denotes the normalized counting measure on the zeros of $p_{n}(\mu ; \cdot)$.

Now we use the well-known formula

$$
\frac{1}{\gamma_{n}(\mu)^{2}}=\inf _{q_{n}(z)=z^{n}+\cdots} \int\left|q_{n}\right|^{2} d \mu
$$


to conclude from (14) that the numbers $1 / \gamma_{n}(\mu)^{1 / n}$ form a sequence bounded away form zero and infinity.

Let now $N \subseteq \mathbf{N}$ be any subsequence of the natural numbers for which the limit $\lim _{n \rightarrow \infty, n \in N} \nu_{n}=\nu$ exists in weak* sense. By a theorem of Widom [Wi] in every compact subset of $\overline{\mathbf{C}} \backslash S(\mu)$ the number of zeros of $p_{n}(\mu ; \cdot)$ is bounded by a bound independent of $n$, hence $\nu$ is supported on the support $S(\mu)$ of $\mu$. We can extract another subsequence from $N$, which we continue to denote by $N$, for which the limit

$$
\lim _{n \rightarrow \infty, n \in N} \log \frac{1}{\gamma_{n}(\mu)^{1 / n}}=-d .
$$

also exist. Then $d$ is a finite number.

It immediately follows from (19) via the principle of descent that

$$
\log \frac{1}{\operatorname{cap}(S(\mu))}-U^{\omega_{S(\mu)}}(z) \leq d-U^{\nu}(z)
$$

for quasi-every $z \in \mathbf{C}$. Since the left-hand side is bounded below on compact subsets of $\mathbf{C}$ and the support of $\nu$ is contained in $S(\mu)$, we can conclude that $\nu$ has finite logarithmic energy, and so we can apply the principle of domination (see the proof in $\S 2.3$ ) to conclude that (22) is true for every $z \in \mathbf{C}$.

Thus,

$$
U^{\nu}(z) \leq U^{\omega_{S(\mu)}}(z)+c
$$

where

$$
c=\log \operatorname{cap}(S(\mu))+d
$$

Now we show that here the equality sign holds for quasi-every $z \in K$. Since sets of capacity zero have $\omega_{S(\mu)}$-measure zero, this will imply together with (23) and Theorem 1 the conclusion

$$
\left.\nu\right|_{K} \geq\left.\omega_{S(\mu)}\right|_{K}
$$

By Theorem 3.1.1 in [StTo] (see also [U12] and (11)) the assumption (13) is equivalent to

$$
\limsup _{n \rightarrow \infty}\left|p_{n}\left(\left.\mu\right|_{K} ; z\right)\right|^{1 / n}=1
$$

for quasi-every $z \in K$. Develop now $p_{n}(\mu ; z)$ into $\left\{p_{k}\left(\left.\mu\right|_{K} ; z\right)\right\}_{k=0}^{\infty}$ :

$$
p_{n}(\mu ; z)=\sum_{k=1}^{n} c_{k, n} p_{k}\left(\left.\mu\right|_{K} ; z\right) \text {. }
$$

Since the $L^{2}\left(\left.\mu\right|_{K}\right)$-norm of the left-hand side is at most 1 , we can see that each coefficient $c_{n, k}$ is at most 1 in absolute value, hence it immediately follows from (19) and (24) that

$$
\limsup _{n \rightarrow \infty}\left|p_{n}(\mu ; z)\right|^{1 / n}=1
$$

for quasi-every $z \in K$. Combining this with the lower envelope theorem we can see that the equality sign holds in (22) for quasi-every $z \in K$, and this is the 
same as our claim that the equality sign holds in (23) for quasi-every $z \in S(\mu)$. This completes the proof of Theorem 3.

The proof of Theorem 5 is analogous. In fact, we can repeat the above proof with $A(\mu)$ replacing $K$ everywhere (see also (16) for the boundedness of the numbers $\log \left(1 / \gamma_{n}(\mu)^{1 / n}\right)$ in the case $\left.c_{\mu}>0\right)$. Then we only have to show that the equality sign holds for quasi-everywhere $z \in A(\mu)$ in (23), which is an immediate consequence of the upper estimate in (19) if we apply the lower envelope principle (recall, that for quasi-every $z \in A(\mu)$ both sides of (19) are 1). This proves Theorem 5 .

3.2.2. Proof of Theorems 4 and 6. We have seen in the proof of Theorem 3 that (with the notations there)

$$
0=d-U^{\nu}(z)
$$

for quasi-every $z \in K$. We put this in the form

$$
U^{\omega_{K}}(z)-\log \frac{1}{\operatorname{cap}(K)} \leq U^{\nu}(z)-d
$$

for quasi-every $z \in K$. Thus, this inequality holds $\omega_{K}$-everywhere, and we get the same inequality for every $z \in \mathbf{C}$ via the principle of domination. Now Theorem 4 follows exactly as in the preceding proof from Theorem 1 if we use (27) for every $z \in \mathbf{C}$ with equality holding for quasi-every $z \in K$ (see (26)).

The proof of Theorem 6 is very similar if we consider that the equality (26) holds true quasi-everywhere on the adjoint set. In fact, let $K \subseteq A(\mu)$ be any fixed compact set. There is an icreasing sequence of compact subsets $K_{n}$ of $A(\mu)$ all containing $K$ such that $\operatorname{cap}\left(K_{n}\right) \nearrow \operatorname{cap}(A(\mu))$. It is known (see [Wy] or [StTo, Appendix IV]) that then $\omega_{K_{n}} \rightarrow \omega_{A(\mu)}$ in weak* sense, hence, using the compactness of $K$ (which implies the upper semicontinuity of its characteristic function), one can easily get that

$$
\omega_{A(\mu)}(K) \geq \limsup _{n \rightarrow \infty} \omega_{K_{n}}(K)
$$

(use that every upper semicontinuous function is the pointwise limit of a decreasing sequence of continuous functions, and apply the monotone convergence theorem).

Now the proof of Theorem 4 gives that the inequality $\left.\nu\right|_{K_{n}} \leq \omega_{K_{n}}$ holds for every $n$, in particular $\nu(K) \leq \omega_{K_{n}}(K)$, and for $n \rightarrow \infty$ we get from (28) the inequality $\nu(K) \leq \omega_{A(\mu)}(K)$. It easily follows from the properties and unicity of the equilibrium measure and from the definition of the adjoint set that $\omega_{A(\mu)}=\omega_{\mu}$, hence we also get $\nu(K) \leq \omega_{\mu}(K)$. This proves Theorem 6 because $K$ can be any compact subset of $A(\mu)$.

3.2.3. Proof of the corollaries. Corollaries 1 and 2 are immediate consequences of Theorems 3 and 4, respectively.

Likewise, the first statement in Corollary 3 follows from Theorem 5 if we note that quasi-every point of any minimal carrier must belong to the adjoint set by the very definition of this set and the equilibrium measure (see (1)-(2)). As for the second statement, let $C$ be a minimal carrier. The first part of the corollary shows that the restriction of $\nu$ to $S \cap C$ is at least as large as that of 
$\omega_{S(\mu)}$. But $S \backslash C$ has zero $\mu$-measure, and hence the assumption on $S$ implies that $\omega_{S(\mu)}(S \backslash C)=0$ also holds, so for any Borel set $B \subseteq S$ we have

$$
\nu(B) \geq \nu(B \cap C) \geq \omega_{S(\mu)}(B \cap C)=\omega_{S(\mu)}(B) .
$$

This completes the proof of Corollary 3.

If we follow the proof of Theorem 6 we can get Corollary 4 from the above observation that quasi-every point of any minimal carrier must belong to the adjoint set $A(\mu)$, and if $K_{n}$ is an increasing sequence of compact subsets of a minimal carrier $C$ with $\operatorname{cap}\left(K_{n}\right) \nearrow \operatorname{cap}(C)=c_{\mu}$, then $\omega_{K_{n}} \rightarrow \omega_{\mu}$ in the weak* topology (see [Wy] or [StTo, Appendix IV]).

This completes our proofs.

\section{ACKNOWLEDGMENT}

We thank Professor Bent Fuglede for providing us with the reference [dVP2] to Theorem 1, as well as the further papers on the subject $[\mathrm{Br}, \mathrm{Gr}, \mathrm{Fu}]$. We have also followed his suggestion concerning Theorem 2, which has simplified our original argument and yielded a sharper version.

\section{REFERENCES}

[Be] A. S. Besicovitch, A general theorem of the covering principle and relative differentiation of additive functions, Proc. Cambridge Philos. Soc. 41 (1945), 103-110.

[Br] M. Brelot, Sur l'allure des fonctions harmoniques et sousharmoniques à la frontière, Math. Nachr. 4 (1950-51), 17-36.

[dVP1] Ch. J. de la Vallée-Poussin: Le potentiel logarithmique, Gauthier-Villars, Paris, 1949.

[dVP2] _ Potentiel et problème généralisé de Dirichlet, Math. Gaz. 22 (1938), 17-36.

[ErFr] P. Erdős and G. Freud, On orthogonal polynomials with regularly distributed zeros, Proc. London Math. Soc. (3) 29 (1974), 521-537.

[ErTu] P. Erdős and P. Turán, On interpolation. III, Ann. of Math. (2) 41 (1940), 510-553.

[Fr] G. Freud, Orthogonal polynomials, Pergamon Press, Oxford, 1971.

[Fu] B. Fuglede, Some properties of the Riesz charge associated with a $\delta$-subharmonic function (manuscript).

[Gr] A. F. Grishin, Sets of regular growth of entire functions, Teor. Funktsii, Funktsional. Anal. i Prilozhen. 40 (1983), 36-47. (Russian)

[La] N. S. Landkof, Foundations of modern potential theory, Grundlehren Math. Wiss., Bd. 190, Springer-Verlag, New York, 1972.

[StTo] H. Stahl and V. Totik, General orthogonal polynomials, Encyclopedia of Mathematics, Vol. 42, Cambridge Univ. Press, New York, 1992.

[Ts] M. Tsuji, Potential theory in modern function theory, Maruzen, Tokyo, 1959.

[U11] J. L. Ullman, On the regular behaviour of orthogonal polynomials, Proc. London Math. Soc. (3) 24 (1972), 119-148.

[U12] _ A survey of exterior asymptotics for orthogonal polynomials associated with a finite interval and a study of the case of general weight measures, Approximation Theory and Spline Functions (S. P. Singh et al., eds.), Reidel, Dodrecht, 1984, pp. 467-478.

[U13] _ Orthogonal polyncmials for general measures. I, Rational Approximation and Interpolation (P. R. Graves-Morris et al., eds.), Lecture Notes in Math., vol. 1105, Springer-Verlag, New York, 1984, pp. 524-528.

[U14] _ Orthogonal polynomials for general measures. II, Polynomes Orthogonaux et Applications (Proc. Bar-le-Duc, 1984, C. Brezinski et al., eds.), Lecture Notes in Math., vol. 1171, Springer-Verlag, New York, 1986, pp. 247-254. 
[Wi] H. Widom, Polynomials associated with measures in the complex plane, J. Math. Mech. 16 (1967), 997-1013.

[Wy] M. F. Wyneken, The potential theory of Borel sets and some applications to the study of orthogonal polynomials (to appear).

Bolyai Institute, Szeged, Aradi v. Tere 1, 6720, Hungary aNd

Department of Mathematics, University of South Florida, Tampa, Florida 33620

E-mail address: totik@math.usf .edu

Department of Mathematics, University of Michigan, Ann Arbor, Michigan 481091003 